\title{
Pacific
}

Journal of

Mathematics

\section{BRAID COMMUTATORS AND VASSILIEV INVARIANTS}

TED STANFORD

Volume $174 \quad$ No. 1 


\title{
BRAID COMMUTATORS AND VASSILIEV INVARIANTS
}

\author{
TED StANFORD
}

\begin{abstract}
We establish a relationship between Vassiliev invariants and the lower central series of the pure braid group, and we use this to construct infinite families of prime knots or links whose invariants match those of a given knot or link up to a given order.
\end{abstract}

\section{Introduction.}

Theorem 1. Let $L$ and $L^{\prime}$ be two links which differ by a braid $p \in P_{k}^{n}$, the nth group of the lower central series of $P_{k}$, the pure braid group on $k$ strands. Let $v$ be a link invariant of order less than $n$. Then $v(L)=v\left(L^{\prime}\right)$.

In Section 1 we will define the order of a link invariant, and also define what it means for two links to differ by a braid $p$. As an example, if $\widehat{x}$ represents the closure of a braid $x$, and $b$ and $p$ are any two braids with the same number of strands, then $\widehat{b}$ and $\widehat{p b}$ differ by $p$. Theorem 1 gives us a way to modify links without changing their invariants up to some order. We shall show that these changes can often be guaranteed to give distinct links.

Falk and Randell proved in [FR] that the intersection of the lower central series of $P_{k}$ is trivial, that is, $\cap_{i=1}^{\infty} P_{k}^{i}=\{1\}$, so that Theorem 1 does not give rise to an easy way of constructing distinct links, all of whose finite-order invariants are equal. We will, however, prove the following in Section 2.

Theorem 2. For any link $L$ and any positive integer $n$ there exist an infinite number of prime, nonsplit, alternating links $L^{\prime}$, (each with the same number of components as $L)$, such that $v\left(L^{\prime}\right)=v(L)$ for any link invariant $v$ of order less than or equal to $n$.

Infinite families of knots whose invariants match up to a given order have been constructed by Ohyama in [O], and also follow from the work of Lin (see [B] and [L2]) and Gusarov (see [Gu2]). However, all of these examples have been composite knots. Recall that a link $L \subset S^{3}$ is composite if there exists a two-sphere $R$ embedded in $S^{3}$ which intersects $L$ in exactly two points, and such that $R$ does not bound a 3-ball $B$ with $B \cap L$ isotopic inside $B$ to an unknotted arc. $L$ is prime if it is not composite. $L$ is split if there exists a 2-sphere $R$ in embedded in $S^{3}$ such that $R$ does not intersect $L$, and $R$ 
does not bound a ball $B$ with $B \cap L=\emptyset . L$ is alternating if it has a diagram $D$ such that as one traverses any component in the diagram, the crossings alternate between over and under.

Knot invariants of finite order are called Vassiliev invariants. A rational Vassiliev invariant is a Vassiliev invariant taking values in $\mathbb{Q}$, the field of rational numbers. It was proved by Birman and Lin (see [BL]) that if the substitution $t=e^{x}$ is made into the Jones polynomial $J_{L}(t)$, then the $i$ th coefficient $v_{i}(L)$ of the resulting power series $J_{L}\left(e^{x}\right)=\sum_{i=0}^{\infty} v_{i}(L) x^{i}$ is a rational Vassiliev invariant of order $i$. It follows directly from this theorem that there are rational Vassiliev invariants of arbitrarilly high order. However, rational Vassiliev invariants form a graded ring (in fact, a Hopf algebra), and there are many finite-order invariants which are products of invariants of lower orders, and these do not provide any power to distinguish links not already available to their lower order factors. A Vassiliev invariant $v$ is called primitive if it is additive under connected sum of knots, that is, $v\left(K_{1} \# K_{2}\right)=v\left(K_{1}\right)+v\left(K_{2}\right)$. It turns out that all the rational Vassiliev invariants are polynomials in the primitive ones. See [B-N], [Gu2].

Corollary 2.1. There are primitive Vassiliev invariants of arbitrarilly high order.

This follows from Theorem 2, and from the fact that the Jones polynomial is never trivial (ie, equal to 1 , its value on the unknot) on a nontrivial alternating knot (see [Mu]). For if there were no primitive Vassiliev invariants of order $\geq n$, then we could construct an alternating knot with all of its finite-order invariants equal to 0 , and therefore with trivial Jones polynomial.

Except when otherwise specified, links in this paper can be considered to be either all oriented or all unoriented. We will denote by $\sigma_{i}$ the standard generator of the braid group $B_{k}$ which switches the $i$ th and $(i+1)$ st strand of the braid with a positive crossing. A tangle diagram will be a link diagram with a single $S^{1}$ boundary, which some of the strands in the diagram may intersect transversely. We will sometimes blur the distiction between braids and braid words, and between links and link diagrams when no ambiguity can result.

The author wishes to thank Professor Joan Birman for many helpful conversations.

\section{Invariants of finite order.}

A link invariant $v$ taking values in an abelian group $A$ is said to have finite order if there exists a positive integer $n$ such that for any link diagram $D$ 
with $n+1$ chosen crossings numbered from 1 to $n+1$

$$
\sum_{i \subseteq\{1,2, \ldots n+1\}}(-1)^{\#(i)} v\left(D_{i}\right)=0
$$

where $\#(i)$ is the cardinality of the set $i$, and $D_{i}$ is obtained from $D=D_{\emptyset}$ by changing each crossing whose number is in $i$. The smallest such $n$ is called the order of $v$, and if there is no such $n$, then $v$ is said to have infinite order. In the case of oriented links, one often defines an invariant $v$ of finite order to be such that there exists a positive integer $n$ satisfying 1.1 , only now the $j$ th crossing of $D_{i}$ is taken to be positive or negative according to whether $j \in i$ or $j \notin i$. It is clear that this is the same as our definition, since the quantity on the left side will differ at most by a sign change between the two cases.

Finite-order invariants of oriented links come in abundance from the Jones polynomial and its generalizations. (See $[\mathbf{B}],[\mathbf{B L}],[\mathbf{G u 1}]$, and $[\mathbf{L 1}]$.) The space $V_{n}$ of rational invariants of order less than or equal to $n$ can be made finite-dimensional either by restricting oneself to links with a fixed number of components or (equivalently, see [S]) by requiring $v$ to be invariant under the addition of a disjoint and unknotted component. Finite-order knot invariants were introduced by Vassiliev in [V] using singularity theory and algebraic topology, and have since been derived, analyzed, and generalized using several other approaches. (See $[\mathbf{B L}],[\mathbf{B}-\mathbf{N}],[\mathbf{G u 2}]$, and $[\mathbf{S}]$.)

By a tangle map $T: B_{k} \rightarrow\{$ link types $\}$ we mean for some fixed $k$ a fixed way of putting a braid $b \in B_{k}$ into a tangle diagram so as to get a link $T(b)$. (This is well-defined because the braid group relations are a subset of Reidemeister moves on link diagrams.) See Figure 1.1. In the case where $T: B_{k} \rightarrow$ \{oriented link types\} then we do not assume that $T$ preserves any orientation on the strands inherited from the braid group, and in fact the strands of $b$ in $T(1)$ need not have their orientations parallel to each other. Given a tangle map $T$ and a link invariant $v$, we denote by $f_{v, T}$ the linear extenion of $v \circ T: B_{k} \rightarrow \mathbb{Q}$ to $\mathbb{Q} B_{k}$.

Two links $L$ and $L^{\prime}$ are said to differ by a braid $b$ if there exists a tangle map $T$ such that $L=T(1)$ and $L^{\prime}=T(b)$. If $L$ and $L^{\prime}$ differ by $b$, then $L^{\prime}$ and $L$ differ by $b^{-1}$, and "differing by $b$ " is not necessarilly a reflexive relation on the set of links. However, we are concerned with links which differ by an element of a particular subgoup of the braid group, so this is not a concern. Note that when $b$ is a pure braid then $L$ and $L^{\prime}$ will have the same number of components.

Consider the ideal $J \in \mathbb{Q} B_{k}$ generated by $\left\{\left(\sigma_{i}-\sigma_{i}^{-1}\right): 0<i<k\right\}$.

Lemma 1.1. Let $v$ be a link invariant of order $n$, and let $T$ be a tangle map. Let $x \in J^{n+1} \subset \mathbb{Q} B_{k}$. Then $f_{v, T}(x)=0$. 
Proof. $J^{n+1}$ is linearly generated by expressions of the form

$$
w_{0}\left(\sigma_{m_{1}}-\sigma_{m_{1}}^{-1}\right) w_{1} \ldots w_{n}\left(\sigma_{m_{n+1}}-\sigma_{m_{n+1}}^{-1}\right) w_{n+1}
$$

where each $\sigma_{m_{j}}$ is one of the standard braid generators and each $w_{j} \in B_{k}$. Consider a diagram for the braid $b=w_{0} \sigma_{m_{1}} w_{1} \sigma_{m_{2}} \ldots \sigma_{m_{n+1}} w_{n+1}$ where each $\sigma_{m_{j}}$ is represented by a single crossing (without any cancellation with $w_{j-1}$ or $w_{j}$ ). Let $D$ be a diagram of $f_{v, T}(b)$ which contains the chosen diagram for $b$, and for $i \subseteq\{1,2, \ldots n+1\}$ let $D_{i}$ be the diagram obtained from $D$ by changing the crossings corresponding to $\sigma_{m_{j}}$ for all $j \in i$. Now it is clear that $f_{v, T}(b)$ is equal to the left-hand side of 1.1 .

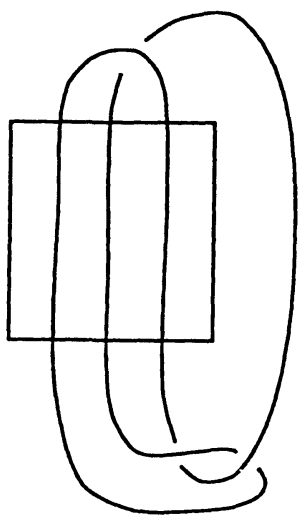

$T(1)$

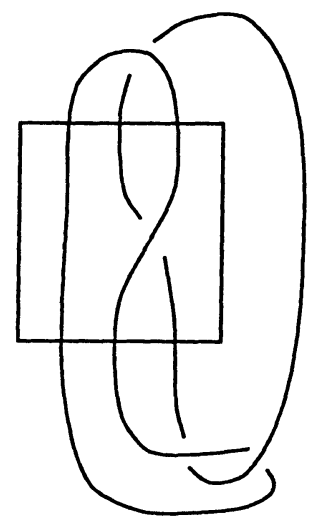

$T\left(\sigma_{2}\right)$

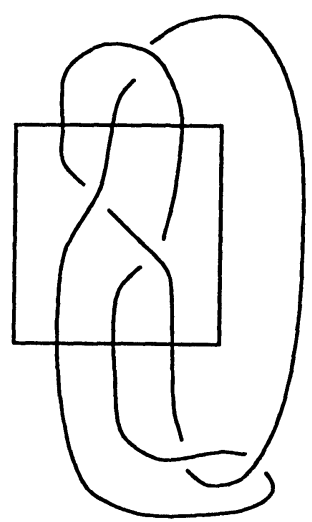

$T\left(\sigma_{1} \sigma_{2}^{-1}\right)$

Figure 1.1

Recall that the lower central series $G=G^{1}, G^{2}, G^{3}, \ldots$ of a group $G$ is given inductively by $G^{n+1}=\left[G, G^{n}\right]$, where $[H, K]=\left\langle x y x^{-1} y^{-1}: x \in\right.$ $H$ and $y \in K\rangle$.

Lemma 1.2. Let $p \in P_{k}^{n}$, the nth group of the lower central series of-the pure braid group $P_{n}$. Then $p-1 \in J^{n}$.

Proof. If $p$ is a pure braid, then $p$ can be "unbraided" by a series of crossing changes from $\sigma_{i}$ to $\sigma_{i}^{-1}$ or vice-versa. Hence $p-1 \in J$. If $p \in P_{k}^{n}$, then

$$
p=\left[p_{1}, q_{1}\right]\left[p_{2}, q_{2}\right] \ldots\left[p_{m}, q_{m}\right]
$$


where $p_{i} \in P_{k}$ and $q_{i} \in P_{k}^{n-1}$. We have $\left[p_{i}, q_{i}\right]-1=\left(p_{i} q_{i}-q_{i} p_{i}\right) p_{i}^{-1} q_{i}^{-1}=\left(\left(p_{i}-1\right)\left(q_{i}-1\right)-\left(q_{i}-1\right)\left(p_{i}-1\right)\right) p_{i}^{-1} q_{i}^{-1} \in J^{n}$ because $p_{i}-1 \in J$ and by induction $q_{i}-1 \in J^{n-1}$.

The ideal $P_{k} \cap J$ is called the augmentation ideal of $P_{k}$, and the group (1+ $\left.J^{n}\right) \cap P_{k}$ is called the $n$th dimension subgroup of $P_{k}$. Lemma 1.2 corresponds to a well-known fact in group theory, that the $n$th dimension subgroup of a group $G$ is a subgroup of $G^{n}$, the $n$th group of the lower cenral series. See [LS].

Theorem 1. Let $L$ and $L^{\prime}$ be two links which differ by a braid $p \in P_{k}^{n}$, Let $v$ be a link invariant of order less than $n$. Then $v(L)=v\left(L^{\prime}\right)$.

Proof. There exists a tangle map $T$ such that $T(1)=L$ and $T(p)=L^{\prime}$. By Lemmas 1.1 and 1.2, $f_{v, T}(p)-f_{v, T}(1)=f_{v, T}(p-1)=0$.

\section{Constructing alternating links.}

Given a subset $S$ of a group $G$, let $W(S)$ be the set of words $x_{1} x_{2} \ldots x_{n}$, for some positive integer $n$ with $x_{i} \in S$. Given any link $L$ and a positive integer $n$, we will to make a series of changes to $L$. Each change will not affect the value $v(L)$ of any invariant $v$ of order less than or equal to $n$, and when we are finished, $L$ will be an alternating link. We will work with a braid word $w \in W\left(\left\{\sigma_{1}, \sigma_{2}, \ldots \sigma_{k-1}, \sigma_{1}^{-1}, \sigma_{2}^{-1}, \ldots \sigma_{k-1}^{-1}\right\}\right)$ which represents a braid whose closure $\widehat{w}$ is $L$. Our objective is to modify $w$ until the odd $\sigma_{i}$ always occur with positive exponent and the even $\sigma_{i}$ always occur with negative exponent, that is, until $w \in W\left(\left\{\sigma_{i}: i\right.\right.$ odd $\} \cup\left\{\sigma_{i}^{-1}: i\right.$ even $\left.\}\right)$. This will ensure that $\widehat{w}$ is an alternating link. See Figure 2.1.

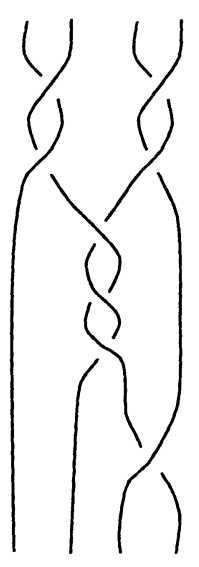

Figure 2.1

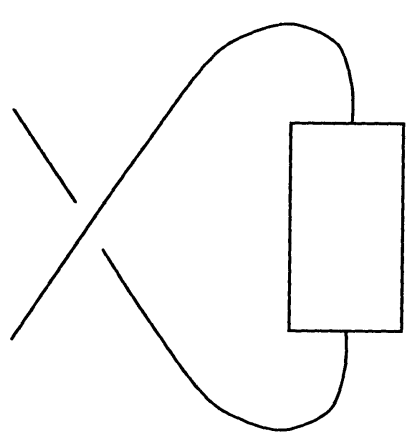

Figure 2.2 
The inspiration for the following lemmas came from [BM]. We shall use the following simplified notation in the proofs. In the braid group $B_{k}$, let $a=\sigma_{i}, b=\sigma_{i+1}$. Then $a b a=b a b$. Let $\bar{x}=(a b a) x(a b a)^{-1}$. Then $\bar{a}=b$ and $\bar{b}=a$, so if $x \in W\left(\left\{a, b^{-1}\right\}\right)$ then $\bar{x} \in W\left(\left\{a^{-1}, b\right\}\right)$, and if $x \in W\left(\left\{a^{-1}, b\right\}\right)$ then $\bar{x} \in W\left(\left\{a, b^{-1}\right\}\right)$. Also if $x \in W\left(\left\{a, b^{-1}\right\}\right)$ then $x^{-1} \in W\left(\left\{a^{-1}, b\right\}\right)$ and if $x \in W\left(\left\{a^{-1}, b\right\}\right)$ then $x^{-1} \in W\left(\left\{a, b^{-1}\right\}\right)$.

Lemma 2.1. For any $n>0$ and $0<i<k-1$, there exist $m>0, r \geq 0$, and $x \in W\left(\left\{\sigma_{i}, \sigma_{i+1}^{-1}\right\}\right)$ such that $t_{i}=\sigma_{i}^{m} x \sigma_{i} \sigma_{i+1}^{-r} \in P_{k}^{n}$.

Proof. Suppose inductively that $t_{i}=a^{m} x a b^{-r} \in W\left(\left\{a, b^{-1}\right\}\right)$ and $t_{i} \in P_{k}^{n}$, with $m>0$ and $r \geq 0$. Form the commutator

$$
\begin{aligned}
{\left[t_{i}, b^{2}\right] } & =a^{m} x a b^{-r} b^{2} b^{r} a^{-1} x^{-1} a^{-m} b^{-2} \\
& =a^{m} x(a b a) a^{-1} b a^{-1} x^{-1} a^{-m+1}(a b a)^{-1} a b^{-1} \\
& =a^{m} x \overline{a^{-1} b a^{-1} x^{-1} a^{-m+1}} a b^{-1} \in W\left(\left\{a, b^{-1}\right\}\right) .
\end{aligned}
$$

To begin the induction we need only note that there are lots of pure braid words in $W\left(\left\{a, b^{-1}\right\}\right)$ that begin with $a$ ( $a^{2}$, for instance).

Remark 2.1. One can also construct words in $W\left(\left\{\sigma_{i}, \sigma_{i+1}^{-1}\right\}\right) \cap P_{k}^{n}$ that begin with $\sigma_{i+1}^{-1}$.

Remark 2.2. Note that $t_{i}$ is represented by a non-trivial alternating word, but we have not proved that $t_{i}$ is a non-trivial braid. It is true that $t_{i} \neq 1$, and this will be seen in the proof of Theorem 2, but we don't need to assume it here.

Lemma 2.2. For any $n>0$ and $0<i<k-1$, there exists $u_{i} \in$ $W\left(\left\{\sigma_{i}, \sigma_{i+1}^{-1}\right\}\right)$ with $u_{i}\left(\sigma_{i} \sigma_{i+1} \sigma_{i}\right) \in P_{k}^{n}$.

Proof. By Lemma 2.1, there exists $t_{i}=a^{m} x a b^{-r} \in W\left(\left\{a, b^{-1}\right\}\right) \cap P_{k}^{n-1}$, with $m>0$ and $r \geq 0$. Form the commutator

$$
\begin{aligned}
{\left[b^{-2}, t_{i}\right] } & =b^{-2} a^{m} x a b^{-r} b^{2} b^{r} a^{-1} x^{-1} a^{-m} \\
& =b^{-2} a^{m} x(a b a) a^{-1} b a^{-1} x^{-1} a^{-m}(a b a)^{-1}(a b a) \\
& =b^{-2} a^{m} x \overline{a^{-1} b a^{-1} x^{-1} a^{-m}}(a b a) .
\end{aligned}
$$

This completes the proof.

Note that $\bar{u}_{i}^{-1} \in W\left(\left\{\sigma_{i}, \sigma_{i+1}^{-1}\right\}\right)$, and $\left(\sigma_{i} \sigma_{i+1} \sigma_{i}\right)^{-1} \bar{u}_{i}^{-1} \in P_{k}^{n}$ because the subgroups of the lower central series are fully invariant.

Theorem 2. For any link $L$ and any positive integer $n$ there exist an infinite number of prime, nonsplit, alternating links $L^{\prime}$, such that $v\left(L^{\prime}\right)=v(L)$ for any link invariant $v$ of order less than or equal to $n$. 
Remark 2.3. The $L$ and $L^{\prime}$ constructed will have the same number of components, so that the theorem remains true if "link" is replaced by "knot".

Proof. Let $L$ be represented as the closure $\widehat{w}$ of some braid word $w$ on $k>2$ strands. To simplify the argument, assume that $k$ is odd. Suppose that $w=z_{1} \sigma_{i}^{-1} z_{2}$, with $i$ odd. Replace $w$ with

$$
z_{1} u_{i}\left(\sigma_{i} \sigma_{i+1} \sigma_{i}\right) \sigma_{i}^{-1}\left(\sigma_{i} \sigma_{i+1} \sigma_{i}\right)^{-1} \bar{u}_{i}^{-1} z_{2}=z_{1} u_{i} \sigma_{i+1}^{-1} \bar{u}_{i}^{-1} z_{2}
$$

where $u_{i}$ is as in Lemma 2.2. By Theorem $1, v(\widehat{w})$ is unchanged by this modification. Repeating the process, we can eliminate all $\sigma_{i}, i$ odd, that occur in $w$ with negative exponent. Similarly, whenever $w=z_{1} \sigma_{i+1} z_{2}$, with $i$ odd, replace $w$ with

$$
z_{1} u_{i}\left(\sigma_{i} \sigma_{i+1} \sigma_{i}\right) \sigma_{i+1}\left(\sigma_{i} \sigma_{i+1} \sigma_{i}\right)^{-1} \bar{u}_{i}^{-1} z_{2}=z_{1} u_{i} \sigma_{i} \bar{u}_{i}^{-1} z_{2} .
$$

Call the result of all these modifications $L^{\prime} . L^{\prime}$ is now given by an alternating diagram $\widehat{w}$. An alternating diagram $D$ is said to be reduced if it contains no crossing as in Figure 2.2. $D$ is said to be prime if there does not exist a simple closed planar curve $C$ that intersects $D$ in exactly two points with $D$ non-trivial (as a diagram) on both sides of $C$. D is connnected if it is connected as a projection onto $\mathbb{R}^{2}$. Menasco proved in [Me] that a reduced, connected, prime alternating diagram represents a prime, non-split link. Let $\alpha$ be the braid word $t_{1} t_{2}^{-1} \ldots t_{k-2} t_{k-1}^{-1}$, where $t_{i}$ is as in Lemma 2.1. Let $\beta$ be the braid word $\sigma_{1} \sigma_{2}^{-1} \ldots \sigma_{k-2} \sigma_{k-1}^{-1}$. It is easy to verify that $\widehat{\beta^{2}}$ is a reduced, connnected, prime, alternating diagram, and that none of these four properties will change if we insert some word $x \in W\left(\left\{\sigma_{i}: i\right.\right.$ odd $\} \cup\left\{\sigma_{i}^{-1}: i\right.$ even $\left.\}\right)$ anywhere into $\beta^{2}$. Since $\alpha^{2} w$ can be built up from $\beta^{2}$ by adding a sequence of such words, it follows that $\widehat{\alpha^{2} w}$ is a reduced, connected, prime, alternating diagram.

Murasugi proved in $[\mathbf{M u}]$ that a reduced alternating link diagram has minimal crossing number, so we can produce an infinite number of distinct $L^{\prime}$ by repeatedly inserting some $t_{i}$ from the lemma into $w$, creating a sequence of reduced, connected, prime, alternating diagrams with more and more crossings.

\section{References}

[B-N] D. Bar-Natan, On the Vassiliev knot invariants, Topology, 34 (1995), 423-472.

[B] J.S. Birman, New points of view in knot theory, Amer. Math. Soc. Bull., 28, No. 2 (1993). 
[BL] J.S. Birman and X.-S. Lin, Knot polynomials and Vassiliev's invariants, Inventiones mathematicae, 111 (1993), 225-270.

[BM] J.S. Birman and W.W. Menasco, Studying links via closed braids III: classifying links which are closed 3-braids, Pacific J. Math., 161 (1993), 25-113.

[FR] M. Falk and R. Randell, Pure braid groups and products of free groups, Contemporary Mathematics, 78 (1988), 217-228.

[Gu1] M.N. Gusarov, A new form of the Conway-Jones polynomial of oriented links, in "Topology of Manifolds and Varieties" (O. Viro, Editor), Amer. Math. Soc., Providence 1994, 167-172.

[Gu2] - On n-equivalence of knots and invariants of finite degree, in "Topology of Manifolds and Varieties" (O. Viro, Editor), Amer. Math. Soc., Providence 1994, 173-192.

[L1] X.-S. Lin, Vertex models, quantum groups and Vassiliev's knot invariants, preprint, Columbia University, 1991.

[L2] - Finite-type link invariants of 3-manifolds, Topology, 33 (1994).

[LS] R.C. Lyndon and P.E. Shupp, Combinatorial group theory, Springer-Verlag, 1977.

[Me] W.W. Menasco, Closed incompressible surfaces in alternating knot and link complements, Topology, 23 (1984), 37-44.

[Mu] K. Murasugi, Jones polynomials and classical conjectures in knot theory, Topology, 26 (1987), 187-194.

[O] Y. Ohyama, Vassiliev invariants and similarity of knots, Amer. Math. Soc Proc., 123 (1995), 287-291.

[S] T. Stanford, Finite-type invariants of knots, links, and graphs, to appear in Topology.

[V] V.A. Vassiliev, Cohomology of knot spaces, in Theory of Singularities and Its Applications (ed. V.I. Arnold), Advances in Soviet Mathematics, Vol. 1, AMS, 1990.

Received August 10, 1993. Research supported in part by the Geometry Center at the University of Minnesota and by the Sloan Foundation.

UNIVERSITY OF CALIFORNIA

BERKELEY, CA 94720

E-mail address: stanford@geom.umn.edu 



\section{PACIFIC JOURNAL OF MATHEMATICS}

Volume $174 \quad$ No. $1 \quad$ May 1996

A distance formula for algebras on the disk

CHRISTOPHER J. BISHOP

Rigidity of isotropic maps

FERNANDO CUKIERMAN

The Schwartz space of a general semisimple Lie group. V. Schwartz

class wave packets

REBECCA A. HERB

Rational polynomials with a $\mathbf{C}^{*}$-fiber

SHULIM KALIMAN

Linear combinations of logarithmic derivatives of entire functions with 195 applications to differential equations

Joseph B. Miles and JoHn Rossi

Factorization problems in the invertible group of a homogeneous $C^{*}$-algebra

N. Christopher PHiLlips

Higher order estimates in complex interpolation theory

RICHARD ROCHBERG

Braid commutators and Vassiliev invariants

TED STANFORD

On the Cauchy problem for a singular parabolic equation 